\title{
Leveraging Machine Perfusion to Ameliorate Geographic Disparities in Organ Allocation
}

\author{
Derek Soled, Cray Noah* \\ Keywords: Organ Allocation, Health Disparities, Lung Transplant, Machine Perfusion, Biotechnology, \\ Resource Allocation
}

\section{INTRODUCTION}

Geographic inequities in access to donor lungs have persisted since the first successful lung transplant in 1983. ${ }^{1}$ With unanswered questions regarding organ preservation and transport in the early days of transplantation, the United Network of Organ Sharing (UNOS) understandably incorporated geography in the allocation algorithm. Today, geography is still the most influential criterion in the lung allocation algorithm. ${ }^{2}$ As a result, patients in urban centers often receive transplants before patients in less-resourced rural areas.

Ex vivo machine perfusion can significantly improve lung procurement and transport, offering longer preservation times before, after, or during transportation. Out-of-hospital perfusion centers, a recent addition to the healthcare field, may increase both the number of lungs available and potentially the distance they can travel. Before the adoption of machine perfusion becomes commonplace, UNOS should direct how to integrate machine perfusion into procurement networks best and shed the antiquated geographical confines that govern allocation today and compromise the ethical standards on which the field was founded.

ANALYSIS

I. The Past: A History of Geographic Disparities in Lung Transplantation

Since the founding of UNOS in 1986, patient geography has been the first filter for all lung procurements. In the early days of the field, implementing these so-called donor service areas, while arbitrarily formed, made sense given the unknowns pertaining to lung preservation and transportation. For almost two decades, donor service areas and time on the waitlist governed lung allocation.

In 1998, after physician protest and advocacy, the U.S. Department of Health and Human Services (HHS) delivered the Final Rule on Organ Transplantation to create a more equitable organ allocation system. Even then, it was not until 2005 that UNOS developed the lung allocation score, a quantitative metric that

\footnotetext{
* Derek Soled and Cray Noah are both MD and MBA Candidates at Harvard Medical School and Harvard Business School
}

(C) 2021 Derek Soled and Cray Noah. This is an open access article distributed under the terms of the Creative Commons Attribution License, which permits unrestricted use, distribution, and reproduction, provided the original author and source are credited. 
considered predicted waitlist survival and transplant benefit. The implementation of the lung allocation score in the U.S. and abroad by Eurotransplant was a success by multiple standards, most importantly reducing waitlist mortality to record lows. ${ }^{3}$ However, a glaring problem remained: the donor service area criterion remained, and arbitrary geographical boundaries continued to govern the distribution of all procured lungs.

Despite the improvements in waitlist mortality, regions with low rates of lung donation, primarily rural areas, have suffered disproportionately. Areas in the lowest quartile of lung availability had an 84 percent increased risk of waitlist death and a 57 percent lower transplantation rate than the top quartile. ${ }^{4}$ In fact, simply moving to an adjacent donor service area a few miles away might double a patient's chances of receiving a lung transplant, significantly more than that patient being bumped into a higher lung allocation score bracket. ${ }^{5}$ That is, driving across an arbitrary border might increase one's chances of receiving a new set of lungs. Unsurprisingly, analysis of data over the last decade shows that donor service areas are independently associated with disparities in access to lung transplants significantly more than any other factor, including gender, ethnicity, diagnosis group, or age.

\section{The Future: Machine Perfusion and Equity in Organ Allocation}

Farther allocation distances are associated with sharper drops in waitlist mortality. A model from Stanford University demonstrates that expanding the existing 250-mile threshold to a 500-mile threshold would decrease waitlist mortality by 21.3 percent; an expansion of 1000 miles would lower it by 31.8 percent. ${ }^{6}$ Since lungs are already more delicate than other solid organs, ${ }^{7}$ an expansion would require better and longer preservation. The answer is already here: machine perfusion.

Ex vivo machine perfusion of organs prior to transplantation has grown remarkably over the past two decades, with recent clinical trial results demonstrating the ability of machine perfusion to resuscitate and assess "marginal" organs prior to transplantation. ${ }^{8}$ Many centers around the U.S. already apply machine perfusion to expand the donor pool and the adoption of machine perfusion as common practice is burgeoning. While the availability of more organs will decrease waiting list mortality, it alone will not address the longstanding geographical disparities. In fact, unless there is deliberate preparation by UNOS, this new biotechnology could very easily exacerbate geographic disparities. It is currently an expensive technology that is exclusive to urban centers with an already high organ availability. Proper foresight before widespread adoption is critical. As machine perfusion will extend the preservation of all solid organs, discussions must start taking place now regarding larger allocation boundaries or even a boundless system altogether.

One concern is that organs resuscitated in this manner will have lower efficacy than organs preserved on ice and rapidly transplanted. Yet, a recent retrospective study from the Toronto group showed that longer perfusion times over 12 hours do not impact patient outcomes, ${ }^{9}$ and some groups have had success with preservation times over 20 hours. ${ }^{10}$ In addition to longer preservation times, machine perfusion can easily be made portable. Data from a recent international pivotal trial using the Organ Care System (OCS) from the Massachusetts-based company TransMedics showed the promising ability of portable machine perfusion to preserve and resuscitate marginal lungs.

Indeed, while much of the attention around machine perfusion has been about its capability to resuscitate marginal organs, its secondary ability, allowing farther transport of lungs, could end geographic disparities in organ allocation. Before it is universally adopted into clinical practice, it is imperative that UNOS acts now to direct hospitals on how to integrate machine perfusion into procurement networks. 
There also must be preemptive policies regarding out-of-hospital perfusion centers. The first and only example thus far is the private corporation Lung Bioengineering, located in Silver Spring, Maryland. This standalone center aims to resuscitate and analyze declined lungs via machine perfusion, shipping viable ones to nearby U.S. transplant centers. The company is currently finishing a phase 2 clinical trial assessing the safety of extending lung preservation times with it. Unless decisive action is taken now, these centers will continue to open exclusively in urban areas surrounded by high-volume centers. To engage in the UNOS organ allocation system, private corporations should be required to distribute to rural and previously under-resourced areas. This could be accomplished by setting up satellite campuses or investing in the necessary infrastructure to preserve and deliver organs far distances portably.

\section{CONCLUSION}

We finally have the tools to extinguish the perennial problem of geographic disparities in organ allocation. Within the next five to ten years, there will be widespread adoption of machine perfusion, both in hospitals and in out-of-hospital perfusion centers. In an already convoluted organ allocation system, it will further complicate organ allocation and will potentially worsen disparities if action is not taken upfront. Establishing regulations to ensure machine perfusion is leveraged in a way that is equitable to all who need solid organ transplants, not only those who live within 250 miles of transplant centers, is crucial. It is necessary for UNOS to be ahead of the curve, mitigate these potential consequences, and reprioritize the ethical principles on which the field was founded. This example should serve as a model for how biotechnology can ameliorate disparities - geographic or otherwise - in scarce resource allocation in healthcare.

\footnotetext{
${ }^{1}$ Lynch, R. J., and R. E. Patzer. 2019. "Geographic inequity in transplant access." Curr Opin Organ Transplant 24 (3): $337-342$. https://doi.org/10.1097/MOT.0000000000000643.
}

${ }^{2}$ Goff, R. R., E. D. Lease, S. Sweet, A. Robinson, and D. Stewart. 2020. "Measuring and Monitoring Equity in Access to Deceased Donor Lung Transplants among Waitlisted Candidates." J Hear Lung Transplant 39 (4): S216.

https://doi.org/10.1016/j.healun.2020.01.847.

${ }^{3}$ Egan, T. M. 2018. "From 6 years to 5 days for organ allocation policy change." J Heart Lung Transplant 37 (5): 675-677. https://doi.org/10.1016/j.healun.2017.12.010.

${ }^{4}$ Benvenuto, L. J., D. R. Anderson, H. P. Kim, J. L. Hook, L. Shah, H. Y. Robbins, F. D'Ovidio, M. Bacchetta, J. R. Sonett, S. M. Arcasoy, and Program From the Columbia University Lung Transplant. 2018. "Geographic disparities in donor lung supply and lung transplant waitlist outcomes: A cohort study." Am J Transplant 18 (6): 1471-1480. https://doi.org/10.1111/ajt.14630.

${ }^{5}$ Kosztowski, M., S. Zhou, E. Bush, R. S. Higgins, D. L. Segev, and S. E. Gentry. 2019. "Geographic disparities in lung transplant rates." Am J Transplant 19 (5): 1491-1497. https://doi.org/10.1111/ajt.15182.

${ }^{6}$ Mooney, J. J., J. Bhattacharya, and G. S. Dhillon. 2019. "Effect of broader geographic sharing of donor lungs on lung transplant waitlist outcomes." J Heart Lung Transplant 38 (2): 136-144. https://doi.org/10.1016/i.healun.2018.09.007.

\footnotetext{
7 Possoz, J., A. Neyrinck, and D. Van Raemdonck. 2019. "Ex vivo lung perfusion prior to transplantation: an overview of current clinical practice worldwide." J Thorac Dis 11 (4): 1635-1650. https://doi.org/10.21037/itd.2019.04.33.

8 Noah, C. V., P. Tratnig-frankl, S. Raigani, C. Cetrulo, K. Uygun, and H. Yeh. 2020. "Moving the Margins: Updates on the Renaissance in Machine Perfusion for Organ Transplantation." Curr Transplant Reports 7 (2): 1-10. https://doi.org/10.1007/s40472-020-00277-z.
} 
Soled And Noah, LeVeraging Machine Perfusion, Voices in Bioethics, Vol. 7 (2021)

9 Yeung, J. C., T. Krueger, K. Yasufuku, M. de Perrot, A. F. Pierre, T. K. Waddell, L. G. Singer, S. Keshavjee, and M. Cypel. 2017. "Outcomes after transplantation of lungs preserved for more than 12 h: a retrospective study." Lancet Respir Med 5 (2): $119-124$. https://doi.org/10.1016/S2213-2600(16)30323-X.

${ }^{10}$ Cypel, M., A. Neyrinck, and T. N. Machuca. 2019. "Ex vivo perfusion techniques: state of the art and potential applications." Intensive Care Med 45 (3): 354-356. https://doi.org/10.1007/s00134-019-05568-3. 